\title{
Calculus of variations involving Caputo-Fabrizio fractional differentiation
}

\author{
Nuno R. O. Bastos ${ }^{1,2, *}$ \\ ${ }^{1}$ Center for Research and Development in Mathematics and Applications (CIDMA), \\ Department of Mathematics, University of Aveiro, Portugal \\ ${ }^{2}$ Department of Mathematics, School of Technology and Management of Viseu, Polytechnic Institute of Viseu, Portugal
}

\begin{abstract}
This paper is devoted to study some variational problems with functionals containing the Caputo-Fabrizio fractional derivative, that is a fractional derivative with a non-singular kernel.
\end{abstract}

Keywords Fractional calculus, calculus of variations, Caputo-Fabrizio fractional derivative

AMS 2010 subject classifications 26A33, 49K05

DOI: $10.19139 /$ soic.v6i1.466

\section{Introduction}

Fractional order calculus is a generalization of the integer order calculus to a real or complex numbers and, nowadays, it plays an important role in various fields: physics (classic and quantum mechanics, thermodynamics, etc.), chemistry, biology, economics, engineering, signal and image processing, and control. Niels Henrik Abel, in 1823, was probably the first to give an application of fractional calculus. Abel applied the fractional calculus in the solution of an integral equation which arises in the formulation of the problem of finding the shape of a frictionless wire lying in a vertical plane such that the time of a bead placed on the wire slides to the lowest point of the wire in the same time regardless of where the bead is placed. This problem is, usually, designated by Tautochrone Problem but sometimes it's also referred as Isochrone Problem. The cycloid is the isochrone as well as the brachistochrone curve. The brachistochrone problem deals with the shortest time of slide and, from mathematical point of view, it marks the begin of the Calculus of Variations. The text is organized as follows. In Section 2 we collect some necessary definitions and results about fractional calculus, fractional calculus of varitions and recall two formulas of integration by parts (Theorem 2). The main results are presented in Section 3. The fundamental problem of calculus of variations that we are considering is then stated in Section 3.1:

$$
J(x)=\int_{a}^{b} L\left(t, x(t),{ }^{C F} D_{a^{+}}^{\alpha} x(t)\right) d t
$$

where ${ }^{C F} D_{a^{+}}^{\alpha} x(t)$ is the left Caputo-Fabrizio fractional derivative (Definition 4). The main results of the paper provide sufficient optimality conditions for the fundamental problem of calculus of variations under appropriate convexity assumptions (see Theorem 4), transversality conditions (see Theorem 5) as well isoperimetric problem and holonomic constraints (see Sections 3.2 and 3.3, respectively). We end up with the conclusion and future work section mentioning some research directions for further work.

\footnotetext{
${ }^{*}$ Correspondence to: Nuno R. O. Bastos (Email: nbastos@estv.ipv.pt). Department of Mathematics, School of Technology and Management of Viseu, Polytechnic Institute of Viseu, 3504-510 Viseu, Portugal
}

ISSN 2310-5070 (online) ISSN 2311-004X (print)

Copyright (C) 2018 International Academic Press 


\section{Fractional calculus}

In a letter to L'Hospital in 1695 Leibniz raised the following question: "Can the meaning of derivatives with integer order be generalized to derivatives with non-integer orders?" L'Hospital was curious about that question and replied by another question to Leibniz: "What if the order will be 1/2? " Leibnitz in a letter dated September 30, 1695 answered: "It will lead to a paradox, from which one day useful consequences will be drawn." The question raised by Leibnitz for a fractional derivative was an ongoing topic for more than 300 years. Many known mathematicians contributed to this theory over the years, among them Liouville, Riemann, Weyl, Fourier, Abel, Lacroix, Leibniz, Grunwald and Letnikov. For more on the subject we refer the reader to [13, 18, 20,23]. There are several definitions of fractional derivatives and fractional integrals, like Riemann-Liouville, Caputo, Riesz, Riesz-Caputo, Weyl, Grunwald-Letnikov, Hadamard, Chen, etc. We will present the definitions of the first two of them (both are fractional derivatives with singular kernel). The Riemann-Liouville fractional derivative (RLFD) was named after the work of Riemann and Liouville and can be defined in the following way:

\section{Definition 1}

Let $x:[a, b] \rightarrow \mathbb{R}$ be a continuos function. The left and the right Riemann-Liouville fractional derivatives of order $\alpha>0$ are defined, respectively, by

$$
{ }^{R L} D_{a^{+}}^{\alpha} x(t)=\frac{1}{\Gamma(n-\alpha)} \frac{d^{n}}{d t^{n}} \int_{a}^{t}(t-\tau)^{n-\alpha-1} x(\tau) d \tau, \quad t \in(a, b],
$$

and

$$
{ }^{R L} D_{b^{-}}^{\alpha} x(t)=\frac{(-1)^{n}}{\Gamma(n-\alpha)} \frac{d^{n}}{d t^{n}} \int_{t}^{b}(\tau-t)^{n-\alpha-1} x(\tau) d \tau, \quad t \in[a, b),
$$

where the $\Gamma$ is the Euler gamma function, $\alpha$ is the order of derivative, and $n=[\alpha]+1$ with $[\alpha]$ being the integer part of $\alpha$.

If $\alpha \geq 1$ is an integer, these fractional derivatives are understood in the sense of usual differentiation, that is,

$$
{ }^{R L} D_{a^{+}}^{\alpha} x(t)=\left(\frac{d}{d t}\right)^{\alpha} x(t) \text { and }{ }^{R L} D_{b^{-}}^{\alpha} x(t)=\left(-\frac{d}{d t}\right)^{\alpha} x(t)
$$

With the objective to avoid some difficulties observed in the utilization of this definition, Michele Caputo introduced, in 1967, the left and right Caputo derivatives, in the following way:

\section{Definition 2}

Let $x:[a, b] \rightarrow \mathbb{R}$ be a continuos function. The left and the right Caputo fractional derivatives of order $\alpha>0$ are defined, respectively, by

$$
{ }^{C} D_{a^{+}}^{\alpha} x(t)=\frac{1}{\Gamma(n-\alpha)} \int_{a}^{t}(t-\tau)^{n-\alpha-1} x^{(n)}(\tau) d \tau, \quad t \in(a, b],
$$

and

$$
{ }^{C} D_{b^{-}}^{\alpha} x(t)=\frac{1}{\Gamma(n-\alpha)} \int_{t}^{b}(-1)^{n}(\tau-t)^{n-\alpha-1} x^{(n)}(\tau) d \tau, \quad x \in[a, b),
$$

where $\alpha$ is the order of derivative, and $n=[\alpha]+1$ with $[\alpha]$ being the integer part of $\alpha$.

There exists a relation between the Riemann-Liouville and the Caputo fractional derivatives:

$$
{ }^{C} D_{a^{+}}^{\alpha} x(t)={ }^{R L} D_{a^{+}}^{\alpha} x(t)-\sum_{k=0}^{n-1} \frac{f^{(k)}(a)}{\Gamma(k-\alpha+1)}(x-a)^{k-\alpha}
$$

and

$$
{ }^{C} D_{b^{-}}^{\alpha} x(t)={ }^{R L} D_{b^{-}}^{\alpha} x(t)-\sum_{k=0}^{n-1} \frac{f^{(k)}(b)}{\Gamma(k-\alpha+1)}(b-x)^{k-\alpha} .
$$


Therefore,

$$
\text { if } x(a)=x^{\prime}(a)=\ldots=x^{(n-1)}(a)=0, \text { then }{ }^{C} D_{a^{+}}^{\alpha} x(t)={ }^{R L} D_{a^{+}}^{\alpha} x(t)
$$

and

$$
\text { if } x(b)=x^{\prime}(b)=\ldots=x^{(n-1)}(b)=0 \text {, then }{ }^{C} D_{b^{-}}^{\alpha} x(t)={ }^{R L} D_{b^{-}}^{\alpha} x(t) .
$$

Recently, in 2015, Caputo and Fabrizio [10] proposed a new derivative of fractional order with a non-singular kernel. This derivative has several interesting properties that are useful for modeling in many branches of sciences. In [6], Atangana and Alqahtani wrote that, for instance, the derivative is able to describe substance heterogeneities and configurations with different scales. Before presenting the definition of the new derivative, we need the following definition:

Definition 3

The Sobolev space of order 1 in $(a, b)$ is defined as

$$
H^{1}(a, b)=\left\{x \in L^{2}(a, b) \mid x^{\prime} \in L^{2}(a, b)\right\},
$$

where $x^{\prime}$ is a weak derivative of $x$.

Now we are in conditions to present the definition of left Caputo-Fabrizio derivative of order $\alpha$.

\section{Definition 4}

Let $\alpha \in(0,1), x$ be a function such that $x \in H^{1}(a, b), a<b$ and $M(\alpha)$ be a normalization function such that $M(0)=M(1)=1$. The left Caputo-Fabrizio derivative of order $\alpha$ is defined as

$$
\left({ }^{C F} D_{a^{+}}^{\alpha} x\right)(t)=\frac{M(\alpha)}{1-\alpha} \int_{a}^{t} x^{\prime}(s) \exp \left(\frac{-\alpha(t-s)}{1-\alpha}\right) d s .
$$

Remark 1

For example, we can consider $M(\alpha)=1-\alpha+\frac{\alpha}{\Gamma(\alpha)}$.

Remark 2

Formula (7) is obtained changing the kernel $(t-\tau)^{-\alpha}$ in (3) with the function $\exp \left(\frac{-\alpha(t-\tau)}{1-\alpha}\right)$ and $\frac{1}{\Gamma(1-\alpha)}$ with $\frac{M(\alpha)}{1-\alpha}$.

Using the idea of Remark 2, Abdeljawad and Baleanu state, in [12], that the right Caputo-Fabrizio fractional derivative is defined by

$$
\left({ }^{C F} D_{b^{-}}^{\alpha} x\right)(t)=-\frac{M(\alpha)}{1-\alpha} \int_{t}^{b} x^{\prime}(s) \exp \left(\frac{-\alpha(s-t)}{1-\alpha}\right) d s
$$

Remark 3

Like the Caputo fractional derivative also Caputo-Fabrizio fractional derivative of a constant is zero.

Recently, Atangana and Goufo [7] developed, the following definitions generated by the modification of (1) and (2).

Definition 5

Let $\alpha \in(0,1), x$ be a function such that $x \in H^{1}(a, b), a<b$ and $M(\alpha)$ be a normalization function such that $M(0)=M(1)=1$. The left Riemann fractional derivative of order $\alpha$ in the Caputo-Fabrizio sense is defined as

$$
\left({ }^{C F R} D_{a^{+}}^{\alpha} x\right)(t)=\frac{M(\alpha)}{1-\alpha} \frac{d}{d t} \int_{a}^{t} x(s) \exp \left(\frac{-\alpha(t-s)}{1-\alpha}\right) d s .
$$

and the right Riemann fractional derivative of order $\alpha$ in the Caputo-Fabrizio sense defined by

$$
\left({ }^{C F R} D_{b^{-}}^{\alpha} x\right)(t)=-\frac{M(\alpha)}{1-\alpha} \frac{d}{d t} \int_{t}^{b} x(s) \exp \left(\frac{-\alpha(s-t)}{1-\alpha}\right) d s .
$$


Similarly to (5) and (6), we find in [12] a relation between Riemann and Caputo type fractional derivatives with non-singular kernels as follows:

Theorem 1 (Proposition 2 of [12])

The following holds:

$$
\begin{aligned}
& \left({ }^{C F} D_{a^{+}}^{\alpha} x\right)(t)=\left({ }^{C F R} D_{a^{+}}^{\alpha} x\right)(t)-\frac{M(\alpha)}{1-\alpha} x(a) \exp \left(\frac{-\alpha(t-a)}{1-\alpha}\right), \\
& \left({ }^{C F} D_{b^{-}}^{\alpha} x\right)(t)=\left({ }^{C F R} D_{b^{-}}^{\alpha} x\right)(t)-\frac{M(\alpha)}{1-\alpha} x(b) \exp \left(\frac{-\alpha(b-t)}{1-\alpha}\right) .
\end{aligned}
$$

Although fractional calculus have various fields of applications, in this paper we are interested in the fractional calculus of variations which deals with optimization of functionals that depends on a fractional operator with a non-singular kernel. For fractional calculus of variations where functional depends on another fractional operators see [1-4,14-17, 19,24,25] and references therein; for computational and numerical aspects see [5,11]. The readers interested in the discrete fractional calculus of variations are refereed to the work of Bastos et al. [8,9]. The theory of the fractional calculus of variations was introduced by Riewe in 1996-1997 [21,22], to deal with nonconservative systems in mechanics. Nowadays, the fractional problems of the calculus of variations is a subject under strong research, mainly, due to its applications in science and engineering.

The following formulas of integration by parts for Caputo-Fabrizio fractional derivatives will be essential for proving results concerning variational problems and were stated and proven in [12].

Theorem 2 (Theorem 2 of [12])

$$
\begin{gathered}
\int_{a}^{b}\left({ }^{C F} D_{a^{+}}^{\alpha} f\right)(t) g(t) d t=\int_{a}^{b} f(t)\left({ }^{C F R} D_{b^{-}}^{\alpha} g\right)(t) d t+\left[\frac{M(\alpha)}{1-\alpha} f(t)\left(\mathbf{e}_{\frac{-\alpha}{1-\alpha}, b^{-}} g\right)(t)\right]_{t=a}^{t=b} . \\
\int_{a}^{b}\left({ }^{C F} D_{b^{-}}^{\alpha} f\right)(t) g(t)=\int_{a}^{b} f(t)\left({ }^{C F R} D_{a^{+}}^{\alpha} g\right)(t)-\left[\frac{M(\alpha)}{1-\alpha} f(t)\left(\mathbf{e}_{\frac{-\alpha}{1-\alpha}, a^{+}} g\right)(t)\right]_{t=a}^{t=b}
\end{gathered}
$$

where

$$
\left(\mathbf{e}_{\frac{-\alpha}{1-\alpha}, b^{-}} g\right)(t)=\int_{t}^{b} \exp \left(\frac{-\alpha(b-t)}{1-\alpha}\right) g(t) d t, t<b
$$

Remark 4

Observe that the left member of equations (13) and (14) contains a Caputo-Fabrizio fractional derivative, while the right member contains a Riemann fractional derivative in the Caputo-Fabrizio sense . However, from Theorem 1 we could present this formulas with only one fractional derivative, although in this case the resulting equations will contain some extra terms.

\section{Main Results}

In this Section we study some problems variational problems with functionals containing the Caputo-Fabrizio fractional derivative, which is a fractional derivative with a non-singular kernel.

\subsection{The Fundamental fractional problem of the calculus of variations}

Given $x \in C^{1}[a, b]$, consider the functional

$$
J(x)=\int_{a}^{b} L\left(t, x(t),{ }^{C F} D_{a^{+}}^{\alpha} x(t)\right) d t,
$$

with the following assumptions: 
1. $L:[a, b] \times \mathbb{R}^{2} \rightarrow \mathbb{R}$ is continuously differentiable with respect to the second and third arguments;

2. given any $x$, the map $t \mapsto{ }^{C F R} D_{b^{-}}^{\alpha}\left(\partial_{3} L\left(t, x(t),{ }^{C F} D_{a^{+}}^{\alpha} x(t)\right)\right)$ is continuous.

Hereafter, given a function $f: A \subseteq \mathbb{R}^{n} \rightarrow \mathbb{R}$, we denote

$$
\partial_{i} f\left(x_{1}, \ldots, x_{n}\right):=\frac{\partial f}{\partial x_{i}}\left(x_{1}, \ldots, x_{n}\right) .
$$

In the next theorem we can find fractional necessary optimality conditions to problem of find local minimizers of (15).

Theorem 3 (Theorem 3 of [12])

Let $x$ be a minimizer of the functional $J$ as in (15), defined on

$$
U=\left\{x \in C^{1}[a, b]: x(a)=x_{a} \quad \text { and } \quad x(b)=x_{b}\right\},
$$

where $x_{a}, x_{b} \in \mathbb{R}$ are fixed. Then, $x$ is a solution for the equation

$$
\partial_{2} L\left(t, x(t),{ }^{C F} D_{a^{+}}^{\alpha} x(t)\right)+{ }^{C F R} D_{b^{-}}^{\alpha}\left(\partial_{3} L\left(t, x(t),{ }^{C F} D_{a^{+}}^{\alpha} x(t)\right)\right)=0, \quad \forall t \in[a, b] .
$$

\section{Definition 6}

Eq. (16) is called the Euler-Lagrange equation associated to the functional $J$. Solutions of this equation are called extremals.

We remark that Eq. (16) gives only a necessary condition. To obtain a sufficient condition, we recall the notion of convex function. Given a function $L(t, x, y)$ continuously differentiable with respect to the second and third arguments, we say that $L$ is convex in $S \subseteq \mathbb{R}^{3}$ if

$$
L\left(t, x+x_{1}, y+y_{1}\right)-L(t, x, y) \geq \partial_{2} L(t, x, y) x_{1}+\partial_{3} L(t, x, y) y_{1} .
$$

Theorem 4

If function $L$ as in (15) is convex in $[a, b] \times \mathbb{R}^{2}$, then each solution of the fractional Euler-Lagrange equation (16) minimizes $J$ in $U$.

Proof Let $x$ be a solution of Eq. (16) and $x+\epsilon h$ be a variation of $x$, with $|\epsilon| \ll 1$ and $h \in C^{1}[a, b]$ with $h(a)=0=h(b)$. Then

$$
\begin{aligned}
& J(x+\epsilon h)-J(x) \\
& =\int_{a}^{b}\left[L\left(t, x(t)+\epsilon h(t),{ }^{C F} D_{a^{+}}^{\alpha} x(t)+\epsilon^{C F} D_{a^{+}}^{\alpha} h(t)\right)-L\left(t, x(t),{ }^{C F} D_{a^{+}}^{\alpha} x(t)\right)\right] d t \\
& \geq \int_{a}^{b}\left[\partial_{2} L\left(t, x(t),{ }^{C F} D_{a^{+}}^{\alpha} x(t)\right) \epsilon h(t)+\partial_{3} L\left(t, x(t),{ }^{C F} D_{a^{+}}^{\alpha} x(t)\right) \epsilon^{C F} D_{a^{+}}^{\alpha} h(h)\right] d t \\
& =\int_{a}^{b}\left[\partial_{2} L\left(t, x(t),{ }^{C F} D_{a^{+}}^{\alpha} x(t)\right)-{ }^{C F R} D_{b^{-}}^{\alpha}\left(\partial_{3} L\left(t, x(t),{ }^{C F} D_{a^{+}}^{\alpha} x(t)\right)\right)\right] \epsilon h(t) d t=0,
\end{aligned}
$$

since $x$ is a solution of (16). Therefore, $x$ is a local minimizer of $J$.

If we do not impose any restrictions on the boundaries or when one the boundary condition is missing, we need to obtain two auxiliary conditions known as transversality conditions.

Theorem 5

Let $x$ be a minimizer of the functional $J$ as in (15). Then, $x$ is a solution for the fractional differential equation

$$
\partial_{2} L\left(t, x(t),{ }^{C F} D_{a^{+}}^{\alpha} x(t)\right)+{ }^{C F R} D_{b^{-}}^{\alpha}\left(\partial_{3} L\left(t, x(t),{ }^{C F} D_{a^{+}}^{\alpha} x(t)\right)\right)=0, \quad t \in[a, b] .
$$

If $x(a)$ is free, then

$$
\frac{M(\alpha)}{1-\alpha} \mathbf{e}_{\frac{-\alpha}{1-\alpha}, b^{-}}\left(\partial_{3} g\left(t, x(t),{ }^{C F} D_{a^{+}}^{\alpha} x(t)\right)\right) \quad \text { at } \quad t=a
$$


If $x(b)$ is free, then

$$
\frac{M(\alpha)}{1-\alpha} \mathbf{e}_{\frac{-\alpha}{1-\alpha}, b^{-}}\left(\partial_{3} g\left(t, x(t),{ }^{C F} D_{a^{+}}^{\alpha} x(t)\right)\right) \quad \text { at } \quad t=b .
$$

Proof Let $x+\epsilon h$ be a variation of $x$, with $|\epsilon| \ll 1$ and $h \in C^{1}[a, b]$. Since $x+\epsilon h$ must belong to the set $U$, the boundary conditions $h(a)=0=h(b)$ must hold. Let $j$ be the function $j$ defined in a neighborhood of zero as

$$
j(\epsilon)=J(x+\epsilon h) .
$$

Since $x$ is a minimizer of $J$, then $\epsilon=0$ is a minimizer of $j$, and so $j^{\prime}(0)=0$. Computing $j^{\prime}(0)$ and using Proposition 2, we get

$$
\begin{gathered}
\int_{a}^{b}\left[\partial_{2} g\left(t, x(t),{ }^{C F} D_{a^{+}}^{\alpha} x(t)\right)+{ }^{C F R} D_{b^{-}}^{\alpha} x(t)\left(\partial_{3} g\left(t, x(t),{ }^{C F} D_{a^{+}}^{\alpha} x(t)\right)\right)\right] h(t) d t \\
+\left[h(t) \frac{M(\alpha)}{1-\alpha} \mathbf{e}_{\frac{-\alpha}{1-\alpha}, b^{-}}\left(\partial_{3} g\left(t, x(t),{ }^{C F} D_{a^{+}}^{\alpha} x(t)\right)\right)\right]_{t=a}^{t=b}=0 .
\end{gathered}
$$

Also, since $x$ is a minimizer, then

$$
\partial_{2} L\left(t, x(t),{ }^{C F} D_{a^{+}}^{\alpha} x(t)\right)+{ }^{C F R} D_{b^{-}}^{\alpha}\left(\partial_{3} L\left(t, x(t),{ }^{C F} D_{a^{+}}^{\alpha} x(t)\right)\right)=0,
$$

for all $t \in[a, b]$. Therefore, using Eq. (17), we obtain

$$
\left[h(t) \frac{M(\alpha)}{1-\alpha} \mathbf{e}_{\frac{-\alpha}{1-\alpha}, b^{-}}\left(\partial_{3} g\left(t, x(t),{ }^{C F} D_{a^{+}}^{\alpha} x(t)\right)\right)\right]_{t=a}^{t=b}=0 .
$$

If $x(a)$ is free, then $h(a)$ is also free and taking $h(a) \neq 0$ and $h(b)=0$, we get

$$
\frac{M(\alpha)}{1-\alpha} \mathbf{e}_{\frac{-\alpha}{1-\alpha}, b^{-}}\left(\partial_{3} g\left(t, x(t),{ }^{C F} D_{a^{+}}^{\alpha} x(t)\right)\right)=0 \quad \text { at } \quad t=a .
$$

The second case is similar.

\subsection{The Isoperimetric Problem}

Historicaly, one of the earliest problems in geometry was the isoperimetric problem, which was considered by the ancient Greeks. The problem is to find, among all closed curves of a given length, the one which encloses the maximum area [26]. One of the oldest problems of this type is Queen Dido's problem. The myth says the Queen Dido landed on the coast of North Africa and asked to the locals for a small piece of land as a temporary place where to stay. She was told that she could have as many land as she could enclose by an oxhide. no such a good answer! But Queen Dido was smart: she cut the oxhide into very thin stips that she used to encircle an entire hill. This is, according to the legend, how Carthage has been founded. The Queen Dido's problem in Calculus of Variation is a good example of isoperimetric problems. In other words, we can state the Queen Dido's problem as the problem of find, among all planar simple closed curves enclosing a fixed area, the one with minimal length. In this section we study the fractional isoperimetric problem where the Lagrangian depends on the left CaputoFabrizio fractional derivative.

Let $l \in \mathbb{R}$ be fixed and $g:[a, b] \times \mathbb{R}^{2} \rightarrow \mathbb{R}$ be a continuously differentiable function with respect to the second and third arguments, such that, for any function $x \in C^{1}[a, b]$, the map $t \mapsto\left(\partial_{3} g\left(t, x(t),{ }^{C F} D_{a^{+}}^{\alpha} x(t)\right)\right)$ is continuous. The integral constraint that we will consider is the following:

$$
I(x)=\int_{a}^{b} g\left(t, x(t),{ }^{C F} D_{a^{+}}^{\alpha} x(t)\right) d t=l .
$$


Theorem 6

Let $x$ be a minimizer of the functional $J$ as in (15), defined on

$$
U=\left\{x \in C^{1}[a, b]: x(a)=x_{a} \quad \text { and } \quad x(b)=x_{b}\right\},
$$

subject to the additional restriction (18). If $x$ is not an extremal of $I$, then there exists a real $\lambda$ such that, defining the function $K:[a, b] \times \mathbb{R}^{2} \rightarrow \mathbb{R}$ by $K=L+\lambda g, x$ is a solution of the equation

$$
\partial_{2} K\left(t, x(t),{ }^{C F} D_{a^{+}}^{\alpha} x(t)\right)+{ }^{C F R} D_{b^{-}}^{\alpha} x(t)\left(\partial_{3} K\left(t, x(t),{ }^{C F} D_{a^{+}}^{\alpha} x(t)\right)\right)=0 .
$$

Proof Consider a variation of $x$ with two parameters $x+\epsilon_{1} h_{1}+\epsilon_{2} h_{2}$, with $\left|\epsilon_{i}\right| \ll 1$ and $h_{i} \in C^{1}[a, b]$ satisfying $h_{i}(a)=0=h_{i}(b)$, for $i=1,2$. Define the functions $i$ and $j$ with two parameters $\left(\epsilon_{1}, \epsilon_{2}\right)$ in a neighborhood of zero as

$$
i\left(\epsilon_{1}, \epsilon_{2}\right)=I\left(x+\epsilon_{1} h_{1}+\epsilon_{2} h_{2}\right)-l, \quad \text { and } \quad j\left(\epsilon_{1}, \epsilon_{2}\right)=L\left(x+\epsilon_{1} h_{1}+\epsilon_{2} h_{2}\right) .
$$

Using Theorem 2 and integration by parts, we get

$$
\begin{aligned}
\frac{\partial i}{\partial \epsilon_{2}}(0,0)=\int_{a}^{b} & \left.\partial_{2} g\left(t, x(t),{ }^{C F} D_{a^{+}}^{\alpha} x(t)\right)+{ }^{C F R} D_{b^{-}}^{\alpha} x(t)\left(\partial_{3} g\left(t, x(t),{ }^{C F} D_{a^{+}}^{\alpha} x(t)\right)\right)\right] h_{2}(t) d t \\
+ & {\left[h_{2}(t) \frac{M(\alpha)}{1-\alpha} \mathbf{e}_{\frac{-\alpha}{1-\alpha}, b^{-}}\left(\partial_{3} g\left(t, x(t),{ }^{C F} D_{a^{+}}^{\alpha} x(t)\right)\right)\right]_{t=a}^{t=b}=0 . }
\end{aligned}
$$

Since $x$ is not an extremal of $I$, there exists some function $h_{2}$ such that $\partial i / \partial \epsilon_{2}(0,0) \neq 0$. By the Implicit Function Theorem, there exists an unique function $\epsilon_{2}(\cdot)$ defined in a neighborhood of zero such that $i\left(\epsilon_{1}, \epsilon_{2}\left(\epsilon_{1}\right)\right)=0$.

On the other hand, $(0,0)$ is a minimizer of $j$, under the restriction $i(\cdot, \cdot)=0$, and we just proved that $\nabla i(0,0) \neq 0$. Appealing to the Lagrange Multiplier Rule, there exists a real $\lambda$ such that $\nabla(j+\lambda i)(0,0)=0$. Differentiating the map $\epsilon_{1} \mapsto j\left(\epsilon_{1}, \epsilon_{2}\right)+\lambda i\left(\epsilon_{1}, \epsilon_{2}\right)$, and putting $\left(\epsilon_{1}, \epsilon_{2}\right)=(0,0)$, we get

$$
\begin{gathered}
\int_{a}^{b}\left[\partial_{2} K\left(t, x(t),{ }^{C F} D_{a^{+}}^{\alpha} x(t)\right)+{ }^{C F R} D_{b^{-}}^{\alpha} x(t)\left(\partial_{3} K\left(t, x(t),{ }^{C F} D_{a^{+}}^{\alpha} x(t)\right)\right)\right] h_{1}(t) d t \\
+\left[h_{1}(t) \frac{M(\alpha)}{1-\alpha} \mathbf{e}_{\frac{-\alpha}{1-\alpha}, b^{-}}\left(\partial_{3} K\left(t, x(t),{ }^{C F} D_{a^{+}}^{\alpha} x(t)\right)\right)\right]_{t=a}^{t=b}=0 .
\end{gathered}
$$

Using the conditions $h_{1}(a)=0=h_{1}(b)$, we prove the desired result.

\section{Remark 5}

We can include the case where $x$ is an extremal of $I$. If we apply the general form of the Lagrange Multiplier Rule, then there are two reals $\left(\lambda_{0}, \lambda\right) \neq(0,0)$ such that $x$ is a solution of the equation

$$
\partial_{2} K_{0}\left(t, x(t),{ }^{C F} D_{a^{+}}^{\alpha} x(t)\right)+{ }^{C F R} D_{b^{-}}^{\alpha} x(t)\left(\partial_{3} K_{0}\left(t, x(t),{ }^{C F} D_{a^{+}}^{\alpha} x(t)\right)\right)=0, \quad \forall t \in[a, b],
$$

where the function $K_{0}:[a, b] \times \mathbb{R}^{2} \rightarrow \mathbb{R}$ is defined by $K_{0}=\lambda_{0} L+\lambda g$.

\subsection{Holonomic Constraints}

Consider the functional $J$ defined by

$$
J\left(x_{1}, x_{2}\right)=\int_{a}^{b} L\left(t, x_{1}(t), x_{2}(t),{ }^{C F} D_{a^{+}}^{\alpha} x_{1}(t),{ }^{C F} D_{a^{+}}^{\alpha} x_{2}(t)\right) d t,
$$

on the space

$$
U=\left\{\left(x_{1}, x_{2}\right) \in C^{1}[a, b] \times C^{1}[a, b]:\left(x_{1}(a), x_{2}(a)\right)=x_{a} \quad \text { and } \quad\left(x_{1}(b), x_{2}(b)\right)=x_{b}\right\},
$$

with $x_{a}, x_{b} \in \mathbb{R}^{2}$ fixed. We are assuming that the Lagrangian verifies the two following conditions 
1. $L:[a, b] \times \mathbb{R}^{4} \rightarrow \mathbb{R}$ is continuously differentiable with respect to its $i$ th argument, for $i=2,3,4,5$;

2. given any function $x$, the maps $t \mapsto{ }^{C F R} D_{b^{-}}^{\alpha}\left(\partial_{i} L\left(t, x(t),{ }^{C F} D_{a^{+}}^{\alpha} x(t)\right)\right)$ are continuous, for $i=4,5$.

We consider in this new variational problem an extra constraint (called in the literature as holonomic constraint). Assume that the admissible functions lie on the surface

$$
g\left(t, x_{1}(t), x_{2}(t)\right)=0
$$

where $g:[a, b] \times \mathbb{R}^{2} \rightarrow \mathbb{R}$ is continuously differentiable with respect to the second and third arguments. For simplicity, we denote

$$
x=\left(x_{1}, x_{2}\right) \quad \text { and } \quad{ }^{C F} D_{a^{+}}^{\alpha} x=\left({ }^{C F} D_{a^{+}}^{\alpha} x_{1},{ }^{C F} D_{a^{+}}^{\alpha} x_{2}\right) .
$$

\section{Theorem 7}

Let $x \in U$ be a minimizer of $J$ as in (20), under the constraint (21). If

$$
\partial_{3} g(t, x(t)) \neq 0 \quad \forall t \in[a, b],
$$

then there is a continuous function $\lambda:[a, b] \rightarrow \mathbb{R}$ such that $x$ satisfies

$$
\partial_{2} L\left(t, x(t),{ }^{C F} D_{a^{+}}^{\alpha} x(t)\right)+{ }^{C F R} D_{b^{-}}^{\alpha}\left(\partial_{4} L\left(t, x(t),{ }^{C F} D_{a^{+}}^{\alpha} x(t)\right)\right)+\lambda(t) \partial_{2} g(t, x(t))=0
$$

and

$$
\partial_{3} L\left(t, x(t),{ }^{C F} D_{a^{+}}^{\alpha} x(t)\right)+{ }^{C F R} D_{b^{-}}^{\alpha}\left(\partial_{5} L\left(t, x(t),{ }^{C F} D_{a^{+}}^{\alpha} x(t)\right)\right)+\lambda(t) \partial_{3} g(t, x(t))=0
$$

on $[a, b]$.

Proof Consider a variation of $x$ of type $x+\epsilon h$, with $|\epsilon| \ll 1$, and $h \in C^{2}[a, b] \times \mathbb{R}^{2}$, satisfying the boundary conditions $h(a)=(0,0)=h(b)$. Since

$$
\partial_{3} g(t, x(t)) \neq 0, \quad \forall t \in[a, b]
$$

by the Implicit Function Theorem, there exists a subfamily of variations satisfying restriction (21), that is, there exists a unique function $h_{2}\left(\epsilon, h_{1}\right)$ such that $\left(x_{1}(t)+\epsilon h_{1}(t), x_{2}(t)+\epsilon h_{2}(t)\right)$ satisfies $(21)$. Hence, for all $t \in[a, b]$, we have

$$
g\left(t, x_{1}(t)+\epsilon h_{1}(t), x_{2}(t)+\epsilon h_{2}(t)\right)=0 .
$$

Differentiating Eq. (23) with respect to $\epsilon$ and putting $\epsilon=0$, we get

$$
\partial_{2} g(t, x(t)) h_{1}(t)+\partial_{3} g(t, x(t)) h_{2}(t)=0 .
$$

Define the function

$$
\lambda(t)=-\frac{\partial_{3} L\left(t, x(t),{ }^{C F} D_{a^{+}}^{\alpha} x(t)\right)+{ }^{C F R} D_{b^{-}}^{\alpha}\left(\partial_{5} L\left(t, x(t),{ }^{C F} D_{a^{+}}^{\alpha} x(t)\right)\right)}{\partial_{3} g(t, x(t))} .
$$

Using equations (24) and (25), we obtain

$$
\lambda(t) \partial_{2} g(t, x(t)) h_{1}(t)=\left[\partial_{3} L\left(t, x(t),{ }^{C F} D_{a^{+}}^{\alpha} x(t)\right)+{ }^{C F R} D_{b^{-}}^{\alpha}\left(\partial_{5} L\left(t, x(t),{ }^{C F} D_{a^{+}}^{\alpha} x(t)\right)\right)\right] h_{2}(t) .
$$

On the other hand, since $x$ is a minimizer of $J$, the first variation of $J$ must vanish:

$$
\begin{gathered}
\int_{a}^{b} \partial_{2} L\left(t, x(t),{ }^{C F} D_{a^{+}}^{\alpha} x(t)\right) h_{1}(t)+\partial_{3} L\left(t, x(t),{ }^{C F} D_{a^{+}}^{\alpha} x(t)\right) h_{2}(t) \\
+\partial_{4} L\left(t, x(t),{ }^{C F} D_{a^{+}}^{\alpha} x(t)\right)^{C F} D_{a^{+}}^{\alpha} h_{1}(t)+\partial_{5} L\left(t, x(t),{ }^{C F} D_{a^{+}}^{\alpha} x(t)\right)^{C F} D_{a^{+}}^{\alpha} h_{2}(t) d t=0 .
\end{gathered}
$$


Integrating by parts, we obtain

$$
\begin{gathered}
\int_{a}^{b}\left[\partial_{2} L\left(t, x(t),{ }^{C F} D_{a^{+}}^{\alpha} x(t)\right)+{ }^{C F R} D_{b^{-}}^{\alpha}\left(\partial_{4} L\left(t, x(t),{ }^{C F} D_{a^{+}}^{\alpha} x(t)\right)\right)\right] h_{1}(t) \\
+\left[\partial_{3} L\left(t, x(t),{ }^{C F} D_{a^{+}}^{\alpha} x(t)\right)+{ }^{C F R} D_{b^{-}}^{\alpha}\left(\partial_{5} L\left(t, x(t),{ }^{C F} D_{a^{+}}^{\alpha} x(t)\right)\right)\right] h_{2}(t) d t=0 .
\end{gathered}
$$

Inserting Eq. (26) into the integral, we get

$$
\int_{a}^{b}\left[\partial_{2} L\left(t, x(t),{ }^{C F} D_{a^{+}}^{\alpha} x(t)\right)+{ }^{C F R} D_{b^{-}}^{\alpha}\left(\partial_{4} L\left(t, x(t),{ }^{C F} D_{a^{+}}^{\alpha} x(t)\right)\right)+\lambda(t) \partial_{2} g(t, x(t))\right] h_{1}(t) d t=0,
$$

and, since $h_{1}$ is arbitrary, we have that $x$ is a solution of the equation

$$
\partial_{2} L\left(t, x(t),{ }^{C F} D_{a^{+}}^{\alpha} x(t)\right)+{ }^{C F R} D_{b^{-}}^{\alpha}\left(\partial_{4} L\left(t, x(t),{ }^{C F} D_{a^{+}}^{\alpha} x(t)\right)\right)+\lambda(t) \partial_{2} g(t, x(t))=0 .
$$

Also, using Eq. (25), we obtain the second condition

$$
\partial_{3} L\left(t, x(t),{ }^{C F} D_{a^{+}}^{\alpha} x(t)\right)+{ }^{C F R} D_{b^{-}}^{\alpha}\left(\partial_{5} L\left(t, x(t),{ }^{C F} D_{a^{+}}^{\alpha} x(t)\right)\right)+\lambda(t) \partial_{3} g(t, x(t))=0 .
$$

The proof is complete.

\section{Theorem 8}

Suppose that the function $L\left(t, x_{1}, x_{2}, y_{1}, y_{2}\right)$ as in (20) is convex in $[a, b] \times \mathbb{R}^{4}, g:[a, b] \times \mathbb{R}^{2} \rightarrow \mathbb{R}$ is continuously differentiable with respect to the second and third arguments, and let $\lambda$ be given by Eq. (25). If $\partial_{3} g(t, x(t)) \neq 0$, for all $t \in[a, b]$, and $x$ is a solution of the fractional Euler-Lagrange equation (22), then $x$ minimizes $J$ in $U$, subject to $(21)$.

Proof If $x+\epsilon h$ is a variation of $x$, then

$$
\begin{aligned}
J(x+\epsilon h)-J(x) \geq & \int_{a}^{b}\left[\partial_{2} L\left(t, x(t),{ }^{C F} D_{a^{+}}^{\alpha} x(t)\right)+{ }^{C F R} D_{b^{-}}^{\alpha}\left(\partial_{4} L\left(t, x(t),{ }^{C F} D_{a^{+}}^{\alpha} x(t)\right)\right)\right] \epsilon h_{1}(t) \\
& +\left[\partial_{3} L\left(t, x(t),{ }^{C F} D_{a^{+}}^{\alpha} x(t)\right)+{ }^{C F R} D_{b^{-}}^{\alpha}\left(\partial_{5} L\left(t, x(t),{ }^{C F} D_{a^{+}}^{\alpha} x(t)\right)\right)\right] \epsilon h_{2}(t) d t
\end{aligned}
$$

Since the variation functions must satisfy the constraint (21), we have the following relation

$$
h_{2}(t)=-\frac{\partial_{2} g(t, x(t)) h_{1}(t)}{\partial_{3} g(t, x(t))}
$$

and using Eq. (25), we deduce

$$
\begin{aligned}
J(x+\epsilon h)-J(x) \geq & \int_{a}^{b}\left[\partial_{2} L\left(t, x(t),{ }^{C F} D_{a^{+}}^{\alpha} x(t)\right)+{ }^{C F R} D_{b^{-}}^{\alpha}\left(\partial_{4} L\left(t, x(t),{ }^{C F} D_{a^{+}}^{\alpha} x(t)\right)\right)\right. \\
& \left.+\lambda(t) \partial_{2} g(t, x(t))\right] \epsilon h_{1}(t) d t
\end{aligned}
$$

which is zero by hypothesis.

\section{Conclusion and future work}

In this paper we have presented variational problems that depend on the Caputo-Fabrizio derivative operator. We exhibit sufficient conditions of optimality for the fundamental problem of the calculus of variations, for problems subject to integral constrains (isoperimetric problems) and for problems subject to holonomic constraints. As future work, it would be interesting to get some real examples/applications for main results of this paper and also make some research to get new properties for the Caputo-Fabrizio derivative operator. 


\section{Acknowledgements}

The author is very grateful to Ricardo Almeida for a careful and thoughtful reading of the manuscript. This work was supported by Portuguese funds through the CIDMA - Center for Research and Development in Mathematics and Applications, and the Portuguese Foundation for Science and Technology (FCT-Fundação para a Ciência e a Tecnologia), within project UID/MAT/04106/2013. The author is also grateful to two anonymous referees for several relevant and stimulating remarks, contributing to improve the quality of the paper.

\section{REFERENCES}

1. R. Almeida, A Caputo fractional derivative of a function with respect to another function, Commun. Nonlinear Sci. Numer. Simul., 44, pp. 460C-481, 2017.

2. R. Almeida, Variational Problems Involving a Caputo-Type Fractional Derivative J. Optim. Theory Appl. 174, no. 1, pp. 276-294, 2017.

3. R. Almeida, R. A. C. Ferreira and D. F. M. Torres, Isoperimetric problems of the calculus of variations with fractional derivatives, Acta Math. Sci. Ser. B Engl. Ed., 32, no. 2, pp. 619-C630, 2012.

4. R. Almeida and A.B. Malinowska, Generalized transversality conditions in fractional calculus of variations, Commun. Nonlinear Sci. Numer. Simul., 18, pp. 443C-452, 2013.

5. R. Almeida, S. Pooseh and D. F. M. Torres, Computational Methods in the Fractional Calculus of Variations, Imp. Coll. Press, London, 2015.

6. A. Atangana and R. T. Alqahtani, Numerical approximation of the space-time Caputo-Fabrizio fractional derivative and application to groundwater pollution equation, Advances in Difference Equations, 156, 13pp, 2016.

7. A. Atangana and E. D. Goufo, Analytical and numerical schemes for a derivative with filtering property and no singular kernel with applications to diffusion, Eur. Phys. J. Plus, pp. 131-269, 2016.

8. N. R. O. Bastos, R. A. C. Ferreira and D. F. M. Torres, Necessary optimality conditions for fractional difference problems of the calculus of variations, Discrete Contin. Dyn. Syst. 29, no. 2, pp. 417-437, 2011.

9. N. R. O. Bastos, R. A. C. Ferreira and D. F. M. Torres, Discrete-time fractional variational problems, Signal Process. 91, no. 3, pp. 513-524, 2011.

10. M. Caputo and M. Fabrizio, A new definition of fractional derivative without singular kernel Progr. Fract. Differ. Appl.,1 (2), pp. 73-85, 2015.

11. S. Jahanshahi and D. F. M. Torres, A simple accurate method for solving fractional variational and optimal control problems, J. Optim. Theory Appl. 174, no. 1, pp. 156-175, 2017.

12. F. Jarad, T. Abdeljawad and D. Baleanu, On fractional derivatives with exponential kernel and their discrete versions, arXiv:1606.07958, 2016.

13. A. A. Kilbas, H. M. Srivastava and J. J. Trujillo, Theory and applications of fractional differential equations, North-Holland Mathematics Studies, 204, Elsevier, Amsterdam, 2006.

14. A. B. Malinowska, T. Odzijewicz and D. F. M. Torres, Advanced methods in the fractional calculus of variations, Springer Briefs in Applied Sciences and Technology, Springer, Cham, 2015.

15. A. B. Malinowska and D. F. M. Torres, Generalized natural boundary conditions for fractional variational problems in terms of the Caputo derivative, Comput. Math. Appl., 59, no. 9, pp. 3110-3116, 2010.

16. A. B. Malinowska and D. F. M. Torres, Fractional calculus of variations for a combined Caputo derivative, Fract. Calc. Appl. Anal 14 , no. 4, pp. 523-537, 2011

17. A. B. Malinowska and D. F. M. Torres, Introduction to the Fractional Calculus of Variations, Imp. Coll. Press, London, 2012.

18. K. S. Miller and B. Ross, An introduction to the fractional calculus and fractional differential equations Wiley, New York, 1993.

19. T. Odzijewicz, A. B. Malinowska and D. F. M. Torres, Fractional variational calculus with classical and combined Caputo derivatives, Nonlinear Anal. 75, no. 3, pp. 1507C-1515, 2012.

20. I. Podlubny, Fractional differential equations, Academic Press, San Diego, CA, 1999.

21. F. Riewe, Nonconservative Lagrangian and Hamiltonian mechanics, Phys. Rev. E (3) 53:2, pp. 1890-1899, 1996

22. F. Riewe, Mechanics with fractional derivatives, Phys. Rev. E (3) 55:3, part B, pp. 3581-3592, 1997

23. S. G. Samko, A. A. Kilbas and O. I. Marichev, Fractional integrals and derivatives, Translated from the 1987 Russian original, Gordon and Breach, Yverdon, 1993.

24. S. G. Samko and B. Ross, Integration and differentiation to a variable fractional order, Integral Transform. Spec. Funct. 1, no. 4, pp. 277-300, 1993

25. D. Tavares, R. Almeida and D. F. M. Torres, Constrained fractional variational problems of variable order. IEEE/CAA J. Automat. Sinica (4) 1, pp. 80-88, 2017.

26. B. van Brunt, The calculus of variations, Universitext, Springer, New York, 2004 